\title{
Investigation of multiple primary cancers in patients with bone and soft tissue primary malignancies: A retrospective, institution-based study
}

\author{
AKIYOSHI SHIMATANI, MANABU HOSHI, NAOTO OEBISU, TADASHI IWAI, \\ NAOKI TAKADA and HIROAKI NAKAMURA \\ Department of Orthopedic Surgery, Osaka City University Graduate School of Medicine, Abeno-ku, \\ Osaka 545-8585, Japan
}

Received March 11, 2020; Accepted June 4, 2020

DOI: $10.3892 / \mathrm{mco} .2020 .2088$

\begin{abstract}
In the aging society in Japan, the occurrence of multiple primary cancers has recently increased due to an increase in life expectancy and increased development in cancer diagnostic technology and improvement in treatment outcomes. However, few reports have focused on multiple primary cancers in patients with bone and soft tissue tumors. The present study aimed to analyze the clinical characteristics of patients with multiple malignancies of the bone and soft tissue. Between April 2008 and April 2017, among 973 patients treated at the Department of Orthopedic Surgery, Osaka City University Hospital, those with multiple primary cancers involving bone and soft tissue were identified. The number of cases with multiple cancers in the present study was 30/973 (3.08\%), including 21 males and 9 females. The median age at diagnosis of patients with bone and soft tissue sarcoma was 73.5 years (range, 7-83 years). There were 22 patients with double cancers, and 5, 2 and 1 patients with triple, quadruple and quintuple cancers, respectively. Colorectal cancer was the most common primary cancer $(\mathrm{n}=9)$. In total, $28.6 \%$ of patients had simultaneous cancer, while $71.4 \%$ of patients had heterochronous cancer. The overall 5-year survival for all patients was $75.6 \%$. The prognosis of patients with double cancer of bone and soft tissue sarcoma was not necessarily poor. However, it is necessary to pay attention to the possibility of secondary malignancy. Therefore, it is essential to be careful and well organized when selecting treatment modalities and to adopt a logistical approach for the care of patients with ongoing multiple malignancies.
\end{abstract}

Correspondence to: Dr Akiyoshi Shimatani, Department of Orthopedic Surgery, Osaka City University Graduate School of Medicine, 1-4-3 Asahi-Machi, Abeno-ku, Osaka 545-8585, Japan E-mail: bpcip920@tcct.zaq.ne.jp

Key words: multiple primary cancers, bone and soft tissue tumors, simultaneous cancer, heterochronous cancer

\section{Introduction}

Multiple primary cancers are defined as the presence of two or more independent primary cancers in an individual (1). The incidence of multiple primary cancers is between 2.3 and 5.2\% of all cancer patients (2-4). Male sex, age, and timing of first cancer diagnosis are significant relevant factors for multiple primary cancers (3). Multiple primary cancers in young men are primarily due to genetic predisposition and secondarily related to the treatment of the first cancer. In contrast, in adults, age at diagnosis is known to be a strong factor in increasing the risk of multiple primary cancers (5). Cross-sectional studies have revealed that patients in their 70s had the highest prevalence of multiple primary cancers (6), at 15\% (7). Age has been reported as a predisposing factor in many malignant tumors (8).

The total number of patients with multiple malignancy is expected to continuously increase due to the increased survival and longevity brought about by the development of cancer treatment. In readiness for the increasing Japan's aging population, clarification on the characteristics of patients with primary malignancies and multiple primary cancers is required. However, few studies have reported the incidence of multiple primary cancers in patients with bone and soft tissue malignancies.

This study aimed to analyze the clinical characteristics of patients with multiple malignancies of the bone and soft tissue. The incidence, frequency, prognosis, and identification of simultaneous or heterochronous cancers were assessed.

\section{Materials and methods}

Materials. This was a retrospective, institution-based study. From our institutional database, 973 patients with bone or soft tissue sarcoma were diagnosed according to the World Health Organization histological classification (9). Of 973 cases, 89 were bone sarcoma and 884 were soft tissue sarcoma. These patients underwent surgery, including biopsies, at our hospital from April 2008 to April 2017. Inclusion criteria were as follows: i) Patients who underwent surgery, including biopsies, at our hospital from April 2008 to April 2017; ii) confirmed 
pathological diagnosis; and iii) patients were diagnosed with two or more independent primary cancers including bone or soft tissue sarcoma. We excluded patients diagnosed with bony metastases and patients with incomplete medical records from the study.

Overall, 30 patients with multiple primary cancers, including bone or soft tissue sarcoma, matched these criteria. The period from the first consultation date to the last consultation date was defined as the follow-up interval.

Methods. Patient's age at sarcoma diagnosis, sex, histological subtype, tumor location, clinical stage based on the AJCC system (7th ed.), simultaneous cancer or heterochronous cancer, history of smoking, family history, and prognosis were reviewed.

In all cases, sarcoma surgery was performed to produce a wide surgical margin, except in atypical lipomatous tumors. Chemotherapy was administered to the patients with osteosarcoma, Ewing sarcoma, rhabdomyosarcoma, and high-grade sarcomas with unresectable and/or distant metastases. The dose and/or intensity of chemotherapy was occasionally reduced according to the patient's performance status (PS), age, and the general condition of the patient. Indications for radiation therapy were as follows: i) High risk of postoperative recurrence with marginal or intra-surgical surgical margin, or ii) surgery could not be performed due to poor patient condition. Informed consent was obtained from all patients to present the data and this study was approved by the institutional review board.

Statistical analysis. The 5-year probability of survival was calculated using Kaplan-Meier analysis (10). Statistical analysis was performed using the Excel Statistics software for Windows (version 2018; SSRI Co., Ltd.).

\section{Results}

Patients and tumor histology. Of 973 patients, 30 (3.08\%) had multiple bone and soft tissue sarcomas. The median age at the time of bone or soft tissue sarcoma diagnosis was 73.5 (range, 7-83) years; 21 patients were male and 9 patients were female. The histological sarcoma types were as follows: Atypical lipomatous tumor $(n=5,16.7 \%)$, undifferentiated pleomorphic sarcoma $(n=5,16.7 \%)$, dedifferentiated liposarcoma $(n=3,10 \%)$, leiomyosarcoma $(n=3,10 \%)$, pleomorphic liposarcoma $(n=3,10 \%)$, chondrosarcoma $(n=2,6.7 \%)$, myxofibrosarcoma $(n=2,6.7 \%)$, and 7 other types of sarcoma $(n=1$, for each of the other 7 types, respectively) (Table I). The average follow-up was 32.6 (range, 1.1-84) months.

Site of primary cancers. Table II shows the data on multiple primary malignancies. Colorectal cancer was the most common primary cancer $(n=9)$, followed by gastric cancer $(n=6)$, malignant lymphoma $(n=3)$, renal cancer $(n=3)$, prostate cancer $(n=3)$, lung cancer $(n=3)$, breast cancer $(n=2)$, and others.

Simultaneous or heterochronous. There were 22 patients with double cancer, including 9 patients with simultaneous cancer and 13 with heterochronous cancer. Five patients had
Table I. Patients and tumor histology.

\begin{tabular}{|c|c|}
\hline Characteristics & Value \\
\hline Total patients & 30 \\
\hline Age, years (range) & $73.5(7-83)$ \\
\hline \multicolumn{2}{|l|}{ Sex, $\mathrm{n}$} \\
\hline Male & 21 \\
\hline Female & 9 \\
\hline \multicolumn{2}{|l|}{ Location, $\mathrm{n}$} \\
\hline Extremity & 20 \\
\hline Trunk & 10 \\
\hline \multicolumn{2}{|l|}{ Histology, n } \\
\hline Atypical lipomatous tumor & 5 \\
\hline Undifferentiated pleomorphic sarcoma & 5 \\
\hline Dedifferentiated liposarcoma & 3 \\
\hline Leiomyosarcoma & 3 \\
\hline Pleomorphic liposarcoma & 3 \\
\hline Chondrosarcoma & 2 \\
\hline Myxofibrosarcoma & 2 \\
\hline Angioleiomyosarcoma & 1 \\
\hline Ewing sarcoma & 1 \\
\hline Fibrosarcoma & 1 \\
\hline Granulocytic sarcoma & 1 \\
\hline Malignant peripheral nerve sheath tumor & 1 \\
\hline Extra-skeletal myxoid chondrosarcoma & 1 \\
\hline Myxoid liposarcoma & 1 \\
\hline \multicolumn{2}{|l|}{ Clinical stage, $\mathrm{n}$} \\
\hline IA & 2 \\
\hline IB & 6 \\
\hline IIA & 2 \\
\hline IIB & 7 \\
\hline III & 6 \\
\hline IV & 7 \\
\hline
\end{tabular}

triple cancer, including 1 patient with simultaneous cancer and 4 patients with heterochronous cancers. Two patients had quadruple heterochronous cancers and one patient was identified with quintuple heterochronous cancers. Overall, 28.6 and $71.4 \%$ of all patients had simultaneous and heterochronous cancers, respectively (Table III).

Clinical information on patients with multiple primary malignancies. Table IV shows the clinical data of those with multiple cancers, including bone and soft tissue malignancies. One osteosarcoma case was suspected to be a secondary cancer associated with Ewing sarcoma treatment (11). The 5-year overall survival for all patients was $75.6 \%$ (Fig. 1).

Case report. An 81-year-old man noticed a mass in his right thigh 8 months prior to his clinical presentation; after a soft tissue tumor was identified at a local hospital, he was referred to our hospital.

Axial T1-weighted and coronal T2-weighted magnetic resonance images showed an iso-intense (T1) and heterogeneously 
Table II. Multiple primary malignancies.

\begin{tabular}{lc}
\hline Primary cancer & Number \\
\hline Colorectal cancer & 9 \\
Gastric cancer & 6 \\
Malignant lymphoma & 3 \\
Renal cancer & 3 \\
Prostate cancer & 3 \\
Lung cancer & 3 \\
Breast cancer & 2 \\
Brain tumor & 1 \\
Bladder cancer & 1 \\
Cervical cancer & 1 \\
Chronic myelogenous leukemia & 1 \\
Endometrial cancer & 1 \\
Oesophageal cancer & 1 \\
Osteosarcoma & 1 \\
Ovarian cancer & 1 \\
Thymic carcinoma & 1 \\
Thyroid cancer & 1 \\
\hline
\end{tabular}

Table III. Simultaneity and heterochrony.

\begin{tabular}{lccr}
\hline Duplication & $\begin{array}{c}\text { Simultaneity, } \\
\mathrm{n}\end{array}$ & $\begin{array}{c}\text { Heterochrony, } \\
\mathrm{n}\end{array}$ & $\begin{array}{c}\text { Total, } \\
\mathrm{n}\end{array}$ \\
\hline Double cancer & 9 & 13 & 22 \\
Triple cancer & 1 & 4 & 5 \\
Quadruple cancer & 0 & 2 & 2 \\
Quintet cancer & 0 & 1 & 1 \\
\hline
\end{tabular}

hyper-intense (T2) mass in the right vastus intermedius muscle (Fig. 2A). Using positron emission tomography-computed tomography, the maximum standardized uptake value (SUV) within the tumor lesion was 16.95, with an abnormal uptake in the colon (Fig. 2B). Undifferentiated pleomorphic sarcoma was diagnosed based on pathological needle biopsy assessment. We performed a wide tumor resection. Postoperatively, colonoscopy revealed colorectal cancer (Fig. 2C). The plan was for a resection of the colorectal cancer; however, CT showed lymph node metastases. Therefore, the best supportive care was selected, given his age, activities of daily living, and general condition. He eventually died of lung metastasis from colorectal carcinoma.

\section{Discussion}

Multiple primary cancers are defined as coexistent cancers that occur simultaneously or sequentially in one individual. In 1887, the first diagnostic criteria for multiple cancer were defined by Billroth (12). Recently, the diagnostic criteria often used are those defined by Warren and Gates in 1932. The criteria are as follows: i) Each tumor individually presents with malignant features at a certain state; ii) each tumor is

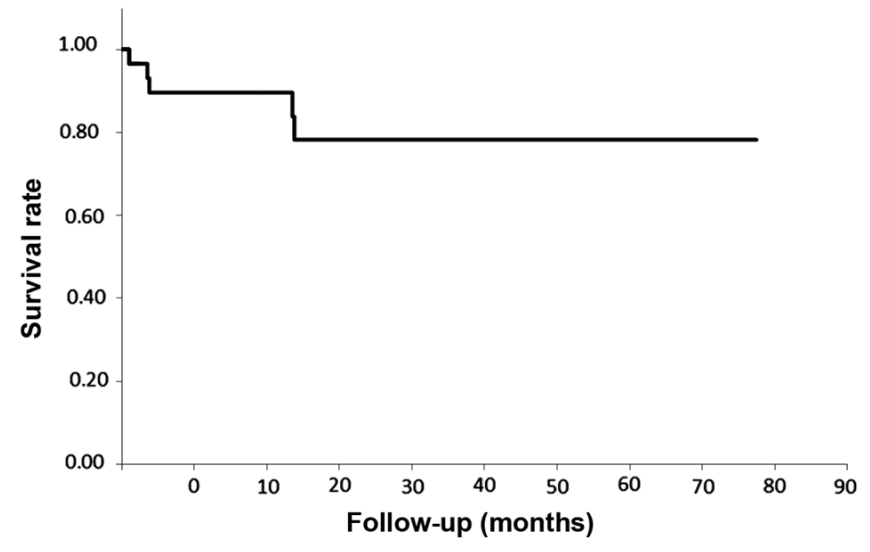

Figure 1. Overall survival of patients with multiple primary cancers. The 5 -year overall survival rate for all patients was $75.6 \%$.

situated apart from the other; and iii) each tumor has been confirmed as not being a metastasis of the other (13).

The occurrence rate of multiple primary cancers is between 2.4 and $5.2 \%$ of all primary malignancies (2-4). While several researches have reported multiple primary cancers, few described multiple primary cancers focusing on bone and soft tissue sarcoma. In this study, we identified only one patient with double sarcoma that was diagnosed as osteosarcoma, being a secondary cancer related to Ewing sarcoma treatment (11). Two studies have analyzed multiple primary cancers with a focus on soft tissue sarcoma $(5,14)$. From the analysis of cancer registry records, 7 of 305 adults with soft tissue sarcoma (2.3\%) developed another primary malignant cancer before or after diagnosis of soft tissue sarcoma (4). Because soft tissue sarcoma was not the focus of the report, the characteristics of multiple primary cancers with soft tissue sarcoma were not explored. Other studies have investigated multiple primary cancers in patients with soft tissue sarcoma and the incidence rate was found to be $9 \%(\mathrm{n}=35 / 406$; with age range, $18-87$ years $)(5)$ and $7.5 \%$ $(n=28 / 375$; with age range, $16-72$ years) (14). Thus, the occurrence of multiple primary malignancies with soft tissue sarcoma differs little from that of other kinds of cancers. In this study, the incidence rate of multiple primary cancers, including bone and soft tissue sarcomas, was 3.08\% (30/973), which was similar to that of multiple primary cancers in all primary cancers.

Cancer that occurs within a year after another cancer was detected is defined as simultaneous cancer, and cancer occurring after 1 year of another is defined as heterochronous cancer; our study adopted this definition. There are some reports of simultaneous multiple primary cancers $(15,16)$. In this study, the rate of simultaneous multiple primary cancers was $28.6 \%$ and the rate of heterochronous multiple primary cancers was $71.4 \%$.

It is necessary to distinguish between hereditary and non-hereditary causes of multiple primary cancers. A hereditary tumor occurs when an abnormality in one gene is inherited, along with other genetic diseases, e.g., Li-Fraumeri syndrome, von Hippel-Lindau disease, and multiple endocrine neoplasia (17). A hereditary tumor should be suspected if the patient had multiple primary cancers at a younger age or has 


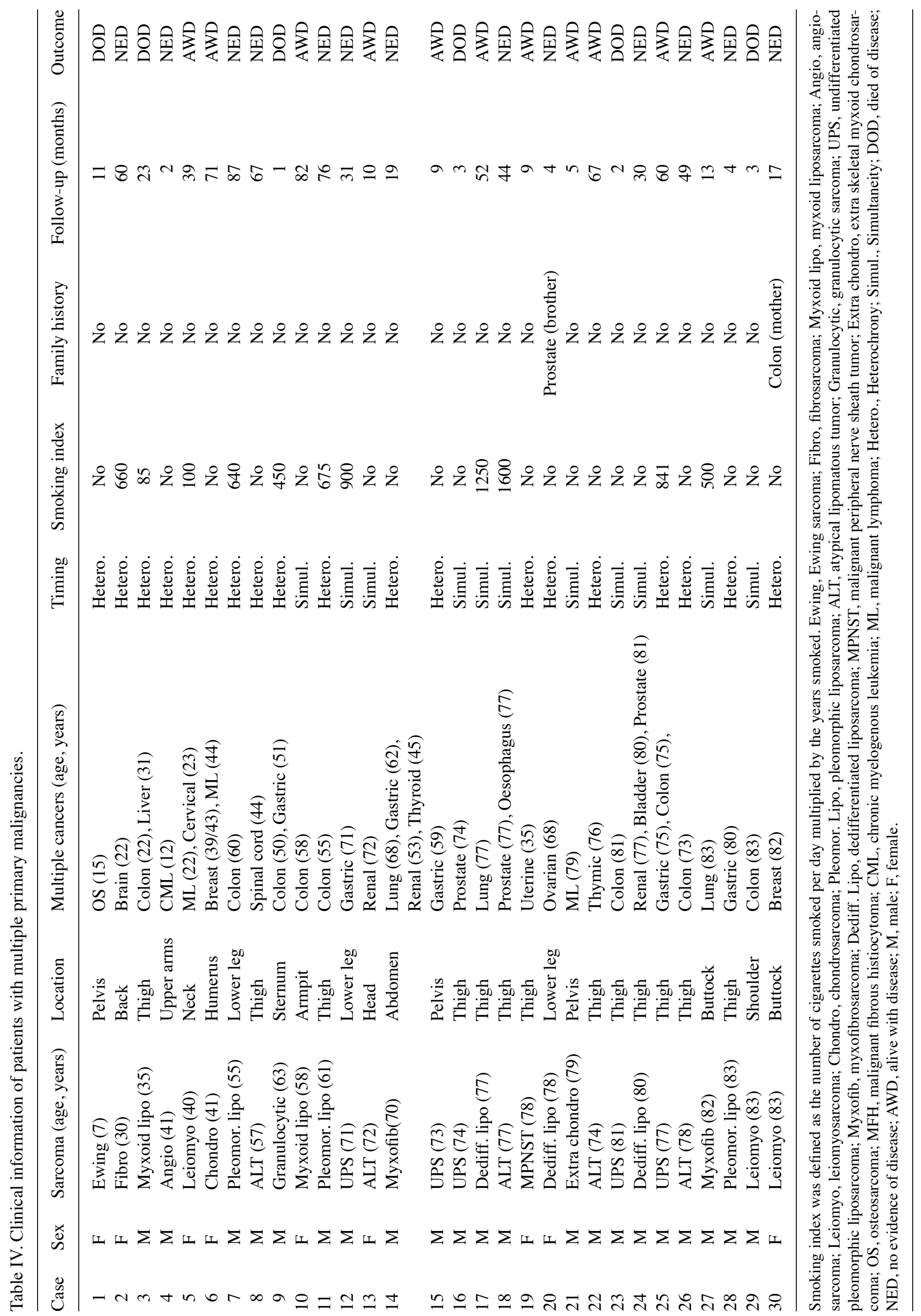



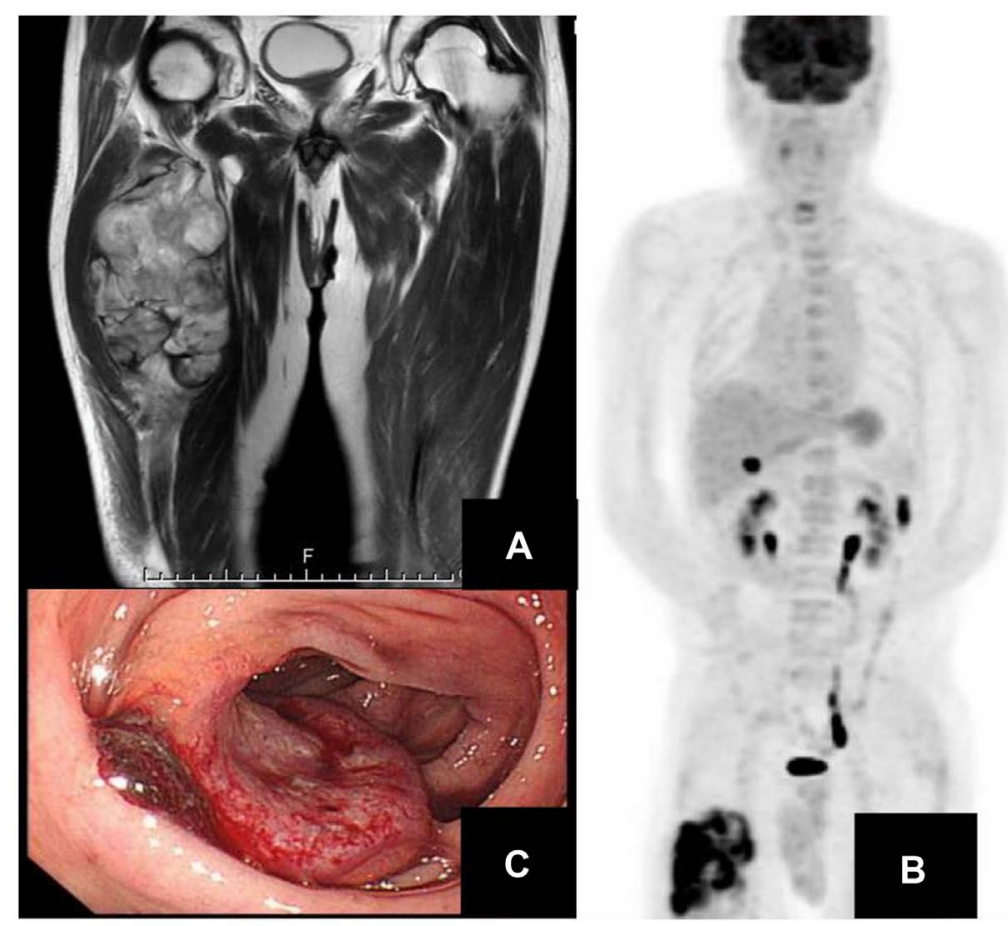

Figure 2. (A) Coronal T2-weighted MRI showing heterogeneous, high intensity of mass. (B) Positron emission tomography-computed tomography showing a right thigh mass (maximum SUV, 16.95) and abnormal colon uptake (maximum SUV, 17.78). (C) Advanced colorectal cancer. SUV, standardized uptake value.

a family history in the same organ. No patient had a relevant family history in this study.

Kozawa et al (18) revealed that the incidence of multiple primary cancers was $23 \%(23 / 100)$ in patients aged $>65$ years with high-grade soft tissue sarcoma. In general, older people have a risk of multiple primary cancers (5). More than $50 \%$ of all cancers occur in individuals aged $>65$ years (19). Furthermore, the accumulation of genetic changes and inaccurate DNA repair become more common with age (20). Advances in diagnostic techniques and multidisciplinary treatment for patients with malignant tumors are contributing factors to the increasing frequency of multiple primary cancers diagnosed in older patients. In this study, the median age was 73.5 years, which was relatively older than that reported in previous studies.

Secondary cancers due to chemotherapy or radiation therapy for primary cancers are also considered multiple primary cancers. The occurrence rate of bone tumors as secondary cancers is $2.8 \%$ within 20 years, and radiation therapy-associated incidence rates depend on the irradiation dose (21). Moreover, alkylating agents are related to the incidence of secondary cancers in a dose-dependent manner (22). In this study, we presented a patient with osteosarcoma as a secondary cancer that occurred in the right femur 7 years post treatment for left-sided Ewing sarcoma of the pelvis. A high dose of alkylating agents was used to treat the Ewing sarcoma (11).

Smoking is a common risk factor for multiple primary malignancies. Cancers associated with smoking include oral, esophageal, lung, bladder, and pancreatic cancers. Point mutations in tumor suppressor gene p53 are familial for smoking-related genetic changes (17). In this study, $36.7 \%$ $(11 / 30)$ of the study participants had a smoking history which was higher than that reported in the national tobacco smoker ratio survey, conducted by the Japan Tobacco Inc. in 2018 (17.9\%) (23).

In this study, colorectal cancer was the most common cancer $(n=9)$ and gastric cancer was the second most common cancer $(n=6)$. The stomach, colon, lungs, and bladder have been listed as organs with the highest frequency of multiple primary malignancies (17). In combined gastric and colorectal cancers, the frequency of microsatellite instability was due to an abnormality in the DNA mismatch mechanism (24).

Patients with duplicated cancer are less likely to develop metastasis, even in stages similar to those of patients with single cancerous lesions, and the prognosis is relatively good (25). Kozawa et al (18) reported that prognosis for patients with multiple cancers with soft tissue sarcoma was not necessarily poor in elderly patients ( $>65$ years). This may be because careful follow-up of the first primary cancer can lead to early detection of secondary cancer. In Japan, based on the registry of 1,769 cases of soft tissue mass in 2015, 5-year overall survival was about $78 \%$ (26). The 5-year overall survival rate for patients with bone sarcoma was $66.9 \%$ from 2008 to 2014, based on data from the Surveillance, Epidemiology, and End Results (SEER) Program (27). In this study, the 5-year survival rate of those with multiple primary cancers, including bone and soft tissue sarcoma, was relatively good (75.6\%). Early detection of multiple primary malignancies in addition to aggressive treatment is considered necessary for a good prognosis.

This study had several limitations. This was a retrospective, institution-based study with a small sample size; in addition, a relatively short follow-up period was used to identify the definitive factors that affected patient prognosis. Furthermore, those with an atypical lipomatous tumor, which is considered an intermediate malignancy, were included. 
In conclusion, as the frequency of multiple primary cancers is expected to increase with the increase in the number of elderly patients, careful follow-up after remission of primary cancer is important. However, the occurrence of multiple primary cancers is not necessarily associated with a poor prognosis.

\section{Acknowledgements}

Not applicable.

\section{Funding}

No funding was received.

\section{Availability of data and materials}

The datasets used and/or analyzed during the current study are available from the corresponding author on reasonable request.

\section{Authors' contributions}

AS, MH and $\mathrm{HN}$ conceived or designed the work. AS, NO, TI and NT collected data. AS, NO, TI and NT performed data analysis and interpretation. AS drafted the article. $\mathrm{MH}$ and HN critically revised the article. HN gave final approval of the version to be published. All authors read and approved the final manuscript.

\section{Ethics approval and consent to participate}

Study approval was obtained from the the Ethical Committee of Osaka City University Graduate School of Medicine, and all investigations were conducted in accordance with the ethical principles of research.

\section{Patient consent for publication}

This study used only information such as medical care information without patient invasion or intervention, and it is not necessary to obtain direct consent from each target patient based on the guidelines established by the government. Information about the conduct of the research, including the purpose of the research, is disclosed, and the opportunity for refusal is guaranteed.

\section{Competing interests}

The authors declare that they have no competing interests.

\section{References}

1. Irimie A, Achimas-Cadariu P, Burz C and Puscas E: Multiple primary malignancies epidemiological analysis at a single tertiary institution. J Gastrointestin Liver Dis 19: 69-73, 2010.

2. Kobayashi Y, Arimoto $H$ and Watanabe S: Occurrence of multiple primary cancer at the National Cancer Center Hospital, 1962-1989. Jpn J Clin Oncol 21: 233-251, 1991.

3. Tsukuma H, Fujimoto I, Hanai A, Hiyama T, Kitagawa T and Kinoshita N: Incidence of second primary cancers in Osaka residents, Japan, with special reference to cumulative and relative risks. Jpn J Cancer Res 85: 339-345, 1994.
4. Ueno M, Muto T, Oya M, Ota H, Azekura K and Yamaguchi T: Multiple primary cancer: An experience at the Cancer Institute Hospital with special reference to colorectal cancer. Int J Clin Oncol 8: 162-167, 2003.

5. Tateishi U, Hasegawa T, Yamamoto S, Yamaguchi U, Yokoyama R, Kawamoto H, Satake M and Arai Y: Incidence of multiple primary malignancies in a cohort of adult patients with soft tissue sarcoma. Jpn J Clin Oncol 35: 444-452, 2005.

6. Adami HO, Bergkvisit L, Krusemo U and Persson I: Breast cancer as a risk factor for other primary malignant diseases. A nationwide cohort study. J Natl Cancer Inst 73: 1049-1055, 1984.

7. Luciani A, Ascione G, Marussi D, Oldani S, Caldiera S, Bozzoni S, Codecà C, Zonato S, Ferrari D and Foa P: Clinical analysis of multiple primary malignancies in the elderly. Med Oncol 26: 27-31, 2009.

8. Balducci L and Ershler WB: Cancer and aging: A nexus at several levels. Nat Rev Cancer 5: 655-662, 2005.

9. Fletcher CDM, Unni KK and Mertens F: World Health Organization classification of tumors. Pathology and genetics of tumors of soft tissue and bone. Lyon: IARC Press, pp341-367, 2002.

10. Kaplan EL and Meier P: Nonparametric-estimation from incomplete observations. J AM Stat Assoc 53: 457-481, 1958.

11. Manabu H, Makoto I, Yamato K, Tokimasa S and Nakamura H: Diagnosis of osteosarcoma in a patient previously treated for Ewing sarcoma. Skeletal Radiol 43: 1319-1324, 2014.

12. Billroth T: Die allgemeine chirurgische pathologie und therapie in 51 Vorlesungen: Ein handbuch fur Studirende und Arzte, 14 Aufl. Berlin, G. Reimer, 1889. (In German)

13. Warren S and Gates O: Multiple primary malignant tumors: A survey of the literature and a statistical study. Am J Cancer 16: 1358-1414, 1932.

14. Merimsky O, Kollender Y, Issakov J, Bickels J, Flusser G, Gutman M, Lev-Chelouche D, Inbar M and Meller I: Multiple primary malignancies in association with soft tissue sarcomas. Cancer 7: 1363-1371, 2001.

15. Singh NJ, Tripathy N, Roy P, Manikantan K and Arun P: Simultaneous triple primary head and neck malignancies: A rare case report. Head Neck Pathol 10: 233-236, 2016.

16. Ikeda Y, Tsukuda M, Ishitoya J, Arai Y, Matsuda H, Katori H, Taguchi T, Yoshida T, Ono M, Sakuma Y, et al: Four cases of simultaneous triple primary cancers of the hypopharynx, esophagus, and stomach. Otolaryngol Head Neck Surg 132: 788-793, 2005.

17. Yuasa Y: Double cancer. Med Prac 17: 698-699, 2000.

18. Kozawa E,Sugiura H,Tsukushi S,Urakawa H,AraiE, FutamuraN, Nakashima H, Yamada Y, Ishiguro N and Nishida Y: Multiple primary malignancies in elderly patients with high-grade soft tissue sarcoma. Int J Clin Oncol 19: 384-390, 2014.

19. Extermann M: Management issues for elderly patients with breast cancer. Curr Treat Options Oncol 5: 161-169, 2004.

20. Anismov VN: The relationship between aging and carcinogenesis: A critical appraisal. Crit Rev Oncol Hematol 45: 277-304, 2003.

21. Tucker MA, D'Angio GJ, Boice JD Jr, Strong LC, Li FP, Stovall M, Stone BJ, Green DM, Lombardi F, Newton W, et al: Bone sarcomas linked to radiotherapy and chemotherapy in children. N Engl J Med 317: 588-593, 1987.

22. Hawkins MM, Wilson LM, Burton HS, Potok MH, Winter DL, Marsden HB and Stovall MA: Radiotherapy, alkylating agents, and risk of bone cancer after childhood cancer. J Natl Cancer Inst 88: 270-278, 1996.

23. Japan Tobacco Inc.'s annual survey. The prevalence of smoking among Japan's adult population. https://www.jti.co.jp/ corporate/enterprise/tobacco/data/smokers/index.html (accessed 11 August 2019).

24. Horii A, Han H, Shimada M, Yanagisawa A, Kato Y, Ohta H, Yasui W, Tahara E and Nakamura Y: Frequent replication errors at microsatellite loci in tumors of patients with multiple primary cancers. Cancer Res 54: 3373-3375, 1994.

25. Baigrie RJ: Seven different primary cancers in a single patient. A case report and review of multiple primary malignant neoplasia. Eur J Surg Oncol 17: 81-83, 1991.

26. Japanese Orthopedic Association Committee on Musculoskeletal Tumor. Soft tissue tumor registry in Japan 2015. Tokyo: National Cancer Center. 2015.

27. National Cancer Institute. SEER Cancer Stat Facts: Bone and Joint Cancer. Bethesda, MD, http://seer.cancer.gov/statfacts/ $\mathrm{html} /$ bones.html. 